\title{
Management of incidental adrenal tumours
}

\author{
Fahmy W F Hanna professor of endocrinology and metabolism ${ }^{1}$, Basil G Issa consultant \\ endocrinologist ${ }^{2}$, Julius Sim professor of healthcare research ${ }^{3}$, Brian Keevil professor of clinical \\ biochemistry ${ }^{4}$, Anthony A Fryer professor of clinical biochemistry ${ }^{5}$
}

${ }^{1}$ Staffordshire University, University Hospital of North Midlands, Stoke-on-Trent, UK; ${ }^{2}$ University Hospital of South Manchester, Manchester, UK; ${ }^{3}$ Keele Medical School, Keele University, Staffordshire, UK; ${ }^{4}$ University Hospital of South Manchester, Manchester, UK; ${ }^{5}$ Institute for Applied Clinical Sciences, Keele University, University Hospital of North Midlands, Stoke-on-Trent, UK

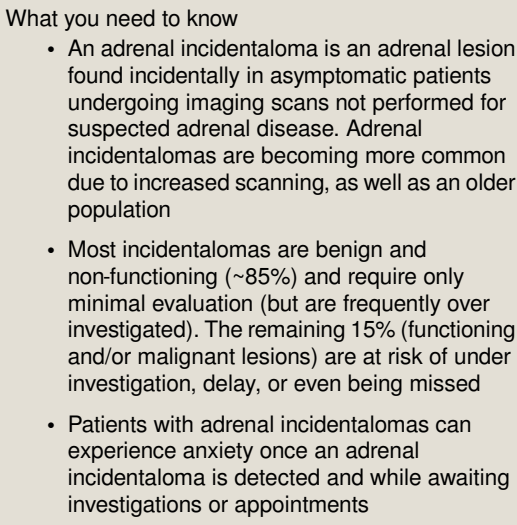

- Most incidentalomas are benign and non-functioning $(\sim 85 \%)$ and require only minimal evaluation (but are frequently over investigated). The remaining $15 \%$ (functioning and/or malignant lesions) are at risk of under investigation, delay, or even being missed

- Patients with adrenal incidentalomas can experience anxiety once an adrenal incidentaloma is detected and while awaiting investigations or appointments

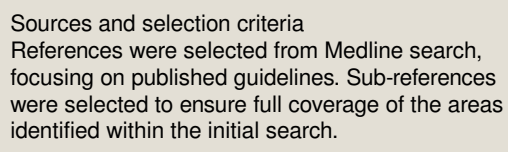

An incidental adrenal tumour, described in this article as an "adrenal incidentaloma," is an adrenal mass discovered during imaging that was not performed for suspected adrenal disease. These are being detected more frequently in an ageing population ${ }^{1}$ through the increased use of computed tomography (eg, computed tomography urogram, or colonoscopy) and magnetic resonance imaging (MRI) scans with enhanced resolution. ${ }^{2}$

This article highlights the management of adrenal incidentalomas, collating recommendations from international guidelines, and is aimed at non-specialists.

\section{What is an adrenal incidentaloma?}

Adrenal incidentalomas are defined as adrenal masses incidentally discovered during imaging that was not performed for suspected adrenal disease. Hence, an adrenal lesion discovered while investigating raised catecholamines would not be classified as an adrenal incidentaloma. Most definitions have restricted the diagnosis to lesions $\geq 10 \mathrm{~mm}^{34}$ unless there are clinical stigmas of adrenal hormone over-production identified after the detection of the lesion that warrant further investigation. In view of variation in diagnostic criteria, referral patterns, and surgical rates, it is challenging to precisely classify the underlying adrenal incidentaloma pathology from published literature. ${ }^{2} \Downarrow$ provides a simplified description of the adrenal gland components, their role, and the consequences of excess hormone production.

\section{Who gets it?}

Prevalence is estimated between $2 \%$ (range 1.0\%-8.7\%) from autopsy studies $^{3-6}$ and $\sim 4 \%$ in radiological studies, rising to $10 \%$ in elderly patients. ${ }^{6-8}$ Prevalence is higher in patients with diabetes, hypertension, or obesity. ${ }^{6}$ Adrenal incidentalomas are rare in children $(<0.04 \%$ of tumours $){ }^{9}$

Most adrenal incidentalomas $(\sim 85 \%)$ are benign, with no hormonal over-production $(\Downarrow) .^{1-10}$ The remaining $\sim 15 \%$, however, are either malignant or associated with hormone over-production (excess cortisol, aldosterone, or catecholamine) and higher morbidity-mortality, thereby requiring rigorous investigation.

The commonest functional abnormality for adrenal incidentalomas is cortisol hyper-secretion, with pheochromocytoma and hyper-aldosteronism being much less common. A 50 year autopsy review of more than 40000 cases revealed pheochromocytomas in $0.13 \%, 76 \%$ of which were only identified after death. ${ }^{11}$ Regardless of whether 
pheochromocytoma had been diagnosed or not, a common cause of death was the development of hypertensive/hypotensive crisis, often precipitated by unrelated surgery.

\section{How are adrenal incidentalomas managed?}

The most recent recommendations from the European Society of Endocrinology (2016) $)^{4}$ on managing adrenal incidentalomas are summarised in $\Downarrow$. Comparisons with guidance from the American Association of Clinical Endocrinologists (2009), ${ }^{12}$ Italian Association of Clinical Endocrinologists (2011), ${ }^{2}$ and Canadian Urological Association (2011), ${ }^{13}$ is available in supplementary table 1 . These recommendations are mostly derived from retrospective studies, case series, and consensus opinion, and they largely agree that

- Screening is necessary for cortisol, aldosterone, and catecholamines hyper-secretion; sex hormones are not routinely measured unless clinically indicated

- Lipid-rich lesions are benign and require no further action

- Indeterminate or lipid-poor lesions require more detailed attention.

\section{Decision making approach}

\section{Is the lesion high risk?}

When assessing patients with newly identified adrenal incidentalomas to determine treatment options and prioritisation, it is important to consider the risk of malignancy, and whether the lesion is hyper-functioning (ie, secreting excessive amounts of adrenal hormones). The risk of malignancy is small in patients not previously known to have cancer. Two large retrospective cohort studies have shown the risk of primary adrenocortical carcinoma to be $4.7 \%$ and $5 \%$, and that of metastasis $0.7 \%$ and $2.5 \% .{ }^{14}$ In a pooled analysis from 26 international studies, the prevalence of adrenocortical carcinomas was $<5 \%$. ${ }^{7}$ There is a good correlation between risk and tumour size: $2 \%$ in lesions $\leq 4 \mathrm{~cm}, 6 \%$ in lesions $4.1-6 \mathrm{~cm}$ and $25 \%$ in lesions $>6 \mathrm{~cm}^{15}$

\section{What is the risk of malignant transformation?}

Size of lesion and its growth over time are key surrogates for malignancy. Of those, size is the most reliable, with $>40 \mathrm{~mm}$ having the highest sensitivity (93\%). ${ }^{14}$ It is unclear what would qualify as a worrying rate of growth, especially as benign adenomas also grow over time. ${ }^{2}$ Retrospective data ${ }^{10}$ showed that, regardless of hormone over-secretion, benign adrenal incidentalomas grew by $10-20 \mathrm{~mm}$ over three years.

\section{If it is not making excess hormones now, could that change in the future?}

Given the limited prospective data, it is not possible to conclusively document the progression towards hyper-secretion. A literature review of longer term follow-up studies ( $>1$ year), ${ }^{7}$ showed that, of 1147 patients with originally non-functional adrenal incidentalomas, 20 (1.7\%) developed hyper-function. The most common evolution is cortisol over-production leading to subclinical hyper-cortisolism. ${ }^{10}$ In a longitudinal follow-up study of 284 consecutive patients with non-secreting adrenal incidentalomas, the cumulative risk of developing subclinical hyper-function was $6.6 \%$ after five years. Those $>30 \mathrm{~mm}$ at diagnosis were associated with a higher risk of hyper-function. ${ }^{16}$ Relative to excess cortisol production, future development of excess aldosterone or catecholamines is unlikely $(<0.1 \%)^{7}$

\section{How might patients with malignant tumours present?}

Most adrenocortical carcinomas are discovered on incidental imaging, and these patients are asymptomatic from an adrenal viewpoint. Malignant adrenal tumours can be functional, and patients might therefore display previously unrecognised features of the corresponding hormone excess, mostly hypercortisolism and/or virilisation. Patients rarely present with signs of feminisation. The sudden onset of virilising clinical features, gynaecomastia, or Cushing's syndrome, might, respectively, point towards an androgen, oestrogen, or cortisol producing adrenocortical carcinoma. ${ }^{17}$ Some patients with adrenocortical carcinomas might present with abdominal or flank pain, abdominal fullness, and occasionally with constitutional symptoms (eg, lethargy, fever, weight loss) secondary to haemorrhage within the tumour.

\section{Investigations to rule out malignancy}

Guidelines agree that no further imaging is necessary where initial scans indicate lipid-rich lesions of less than 10 Hounsfield units (HU) (the measure of tissue density) in patients presenting with no known malignancy; these are classified as benign adenomas. ${ }^{4}$ Unfortunately, about one third of lesions will be lipid-poor (>10 HU) and therefore it is difficult to exclude malignancy. In these cases, a contrast enhanced triphasic adrenal computed tomography scan should be undertaken, with the calculation of the absolute and relative washout percentages. An absolute washout $>60 \%$ or relative washout $>40 \%$ suggest a benign lesion with excellent sensitivity $(88 \%-96 \%)$ and specificity $(96 \%-100 \%){ }^{18} \Downarrow$ shows computed tomography examples of (a) lipid-rich and (b) lipid-poor lesions.

MRI is similarly effective, with the advantage of no radiation exposure and avoiding the use of iodine based contrast agents. ${ }^{19}$ MRI is a safer option for younger patients and if repeated imaging is required (eg, adrenal incidentalomas confirmed to be pheochromocytomas with underlying genetic mutations; these constitute $15 \%-20 \%$ of all catecholamine-secreting tumours).

2-Deoxy-2-[fluorine-18] fluoro-D-glucose positron emission tomography/computed tomography $\left({ }^{18}\right.$ F-FDG PET/CT), a technique that combines nuclear medicine with cross sectional imaging, ${ }^{20}$ is particularly useful in detecting metastatic disease in patients with primary adrenal cancer or secondaries from other extra-adrenal malignancies. ${ }^{21} 22$ The newest imaging option is ${ }^{68} \mathrm{Ga}$-dotatate PET, which has a higher specificity for pheochromocytoma compared with FDG PET/CT. ${ }^{23}$

A recent meta-analysis of 32 observational studies including 2174 patients concluded that adrenal biopsy is useful in the diagnosis of adrenal metastasis in patients with known extra-adrenal malignancy. The authors concluded that adrenal biopsy should only be performed if the findings would alter patient management and after biochemical exclusion of a phaeochromocytoma. ${ }^{24}$

There are no well established sensitive biochemical markers for the diagnosis of adrenocortical carcinoma, or indeed adrenal metastasis. A study including 45 individuals with adrenocortical carcinomas and 102 patients with adrenocortical adenomas showed that detailed serum and 24 hour urinary steroid profiling had a sensitivity and specificity of $90 \%$ in differentiating benign adrenal adenomas from adrenocortical carcinomas. Further work is required to clarify whether such profiling could be useful for monitoring. ${ }^{25}$ 


\section{Hormone over-production in adrenal incidentaloma}

Previously unrecognised clinical features of Cushing's syndrome, pheochromocytoma, or hyper-aldosteronism are suggestive of functional lesions. In reality, however, most adrenal incidentalomas are identified in patients who are asymptomatic from an adrenal viewpoint.

All guidelines agree that hormonal evaluation by specialists is crucial in all adrenal incidentalomas to exclude the three major conditions associated with functional adrenal incidentaloma:

Glucocorticoid excess-There is consensus that the $1 \mathrm{mg}$ overnight dexamethasone suppression test should be the initial screening test. Values $<50 \mathrm{nmol} / \mathrm{L}(1.8 \mu \mathrm{g} / \mathrm{dL})$ are normal, while those $>138 \mathrm{nmol} / \mathrm{L}(5 \mu \mathrm{g} / \mathrm{dL})$ indicate autonomous cortisol production (defined as subclinical Cushing's in the absence of clinical features). However, there is lack of agreement on the interpretation of values between $50 \mathrm{nmol} / \mathrm{L}$ and $138 \mathrm{nmol} / \mathrm{L}$ in asymptomatic patients. European guidelines define this as "possible autonomous cortisol secretion," and US, Italian, and Canadian guidelines acknowledge its significance, despite the absence of unequivocal data on the long term consequences. Another potentially useful test for autonomous cortisol secretion is serum dehydroepiandrosterone sulphate, which is reduced in cortisol hyper-secretion because of adrenocorticotrophic hormone suppression. The dehydroepiandrosterone sulphate ratio (measured as dehydroepiandrosterone sulphate divided by the lower reference limit) has been shown to have good sensitivity and specificity in identifying adenomas with autonomous cortisol secretion. ${ }^{26}$

Mineralocorticoid excess - The guidelines agree about screening using plasma aldosterone (ng/dL)/renin $(\mathrm{ng} / \mathrm{mL} / \mathrm{h})$ ratio only in the presence of hypertension or unexplained hypokalaemia. Values $>20$ (US guidelines) and $>30-50$ (Italian guidelines) require further confirmatory tests. The sample should be taken in the morning, two hours after getting out of bed, and after 5-15 minutes' rest. A careful assessment of the patient's antihypertensive medication should be undertaken (to evaluate potential impact on the ratio) and some might need to be stopped before the test. ${ }^{27}$ Poor adherence to sampling criteria often leads to challenges in interpretation. ${ }^{27}$

Catecholamine excess-All guidelines highlight the importance of screening for pheochromocytoma with plasma or urinary metanephrines, whether or not the patient is hypertensive, as some patients with pheochromocytomas are asymptomatic. Excluding a functioning pheochromocytoma is crucial, as surgery without adequate $\alpha$ then $\beta$ blockade might be seriously detrimental to the patient. ${ }^{28} \mathrm{~A}$ value greater than threefold above the upper reference range limit confirms the diagnosis. Lower values might indicate a false positive test, but pheochromocytoma should be considered in patients with borderline values and indeterminate computed tomography imaging features. ${ }^{4}$

In addition to the above investigations, patients with clinical features of virilisation or gynaecomastia also need assessment of androgen profile and oestrogen levels, respectively. ${ }^{13}$

Steroid precursors and urinary steroid profiles have also been suggested, though these tests are labour intensive, expensive, and not widely available. Evidence for their use in the diagnosis of adrenocortical carcinoma is limited.
Some tumours secrete more than one hormone. Patients with lesions causing both primary hyper-aldosteronism and autonomous cortisol production have been reported. ${ }^{29} 30$

\section{Follow-up}

Follow-up of adrenal incidentalomas is dependent on the characteristics of the original lesion. Current guidelines agree that patients with benign lesions that are non-functional require no further follow-up. ${ }^{4}$ Supplementary table 2 outlines variations on this. However, prospective research studies should be undertaken, as these will strengthen (or challenge) these recommendations.

\section{Present challenges and future direction}

Despite current guidance, there remain two major challenges:

- Most patients with adrenal incidentalomas are not referred to relevant specialists (endocrinologists/diabetologists), so there is potential to miss functional or malignant lesions. That said, investigating every lesion could overload any health system. Therefore, an evidence based, streamlined approach is required to ensure clinically effective and prompt evaluation, while avoiding unnecessary investigations and clinic appointments. We are developing an electronic adrenal incidentaloma management system linked to the latest guidance. ${ }^{4}$ This will provide more streamlined and timely management by bringing all the key information together electronically and guiding the management process, shortening the hands-on time for healthcare professionals. This increased efficiency will facilitate the management of all adrenal incidentaloma cases, including those currently missed thereby enhancing patient safety.

- It is important to recognise that detection of adrenal incidentaloma is associated with substantial patient anxiety (unexpected nature, lack of standardised management pathway, often poor communication from non-specialists); delay in decision making should be avoided (see Tips for the non-specialist).

Future developments in imaging and biochemical marker (eg, steroid metabolites) assays might help differentiate benign from malignant lesions and avoid unnecessary surgery.

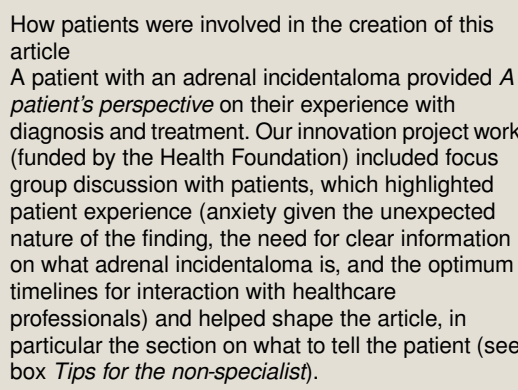


A patient's perspective

\section{Diagnosis}

"Before diagnosis, I suffered sudden fluctuations in body temperature and palpitations. My doctor sent me for blood tests, which were all normal, and an ultrasound scan. I was informed that the scan showed they had found a lump and I was being referred to a consultant.

"Once under hospital care, I felt totally swept along with different scans and tests. I was informed that I had an uncommon adrenal lump that can be serious. The experience was overwhelming; I took in very little information. I did my own research, but found the internet confusing."

\section{Removal and beyond}

"Blood pressure tablets were introduced in the weeks before the surgery. While encouraging talks kept us going; with hindsight, I realise that the operation was risky. I was lucky to have a good outcome, going home one week after surgery. I feel $100 \%$ better and no longer get palpitations or temperature fluctuations. "Family members were tested and none was found to be carrying the broken gene; a relief to everyone. "My care during and after diagnosis was excellent. My advice to other patients is to trust the healthcare professionals, feel free to ask questions, and ensure internet information is from credible websites."

\section{Tips for the non-specialist}

\section{What to say to patients}

- The scan that we arranged for you showed a swelling on the adrenal gland. The adrenal gland is a small but important gland that sits on top of the kidney. It secretes a group of hormones that are important for our wellbeing

- We are increasingly detecting these swellings because our scanners are becoming better

- We know that these swellings could have been present for many years. They are likely to stay largely unchanged with no harm to you. We are reassured by small swellings $(<4 \mathrm{~cm})$. However, we still need to do some blood tests/investigations to make sure all is well

- We will therefore refer you to an endocrinologist/diabetologist (hormone specialist) to do some tests. They will discuss the results and scans in a meeting with other specialists, such as endocrinologists, radiologists, surgeons, and clinical biochemists

\section{When to reassure patients}

Patients will be anxious while awaiting the conclusion of the investigations required by the hormone specialist. Hence, any investigations need to be timely. Lipid-rich swelling less than $4 \mathrm{~cm}$ in diamete with no evidence of hormone over-production will require no further intervention, and patients should be reassured as soon as the biochemical results are available $^{4}$

\section{When to refer to the endocrinology team 4}

- Refer all detected adrenal incidentalomas

- Where there is evidence of excess aldosterone or cortisol, refer within 6-8 weeks

- Where there are large (or increasing in size)/lipid-poor lesions (suspected malignancy) or evidence of excess catecholamines, refer within two weeks

\section{Monitoring and prognosis}

Lipid-rich swelling less than $4 \mathrm{~cm}$ in diameter with no evidence of hormone over-production requires no further action. Other lesions require specialist input

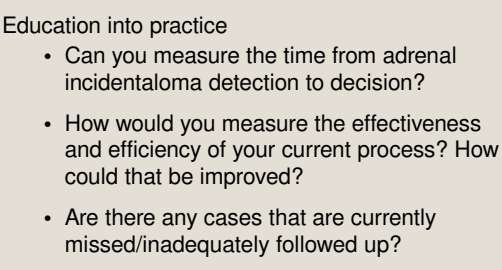

Acknowledgments The authors wish to express their gratitude to the patient who participated in the creation of this article for agreeing to share their experience, having been identified with an adrenal incidentaloma. We also thank Cherian George for kindly identifying and providing the scan images included (for which patient consent was obtained).

Competing interests All authors have completed the Unified Competing Interest form (available on request from the corresponding author) and declare no support from any organisation for the submitted work; no financial relationships with any organisations that might have an interest in the submitted work in the previous three years, and no other relationships or activities that could appear to have influenced the submitted work.

Contributions and guarantor FWFH conceived the original idea and wrote the first draft of the manuscript, particularly the clinical and natural history sections of the manuscript. BGI provided a critical appraisal of alternative guidelines. JS and BK provided an independent critical review of the methodological and biochemical aspects of the manuscript, respectively. AAF wrote the biochemical components and critically reviewed the manuscript as a whole. All authors reviewed and approved the final version of the manuscript. FWFH is the guarantor of the work. Provenance and peer review: commissioned; externally peer reviewed. Patient consent obtained (for $\Downarrow$, which shows CT scans from two patients with adrenal incidentaloma).

1 YoungWFJr. Management approaches to adrenal incidentalomas. A view from Rochester, Minnesota. Endocrinol Metab Clin North Am2000;29:159-85, doi:10.1016/S0889-8529(05)70122-510732270

2 TerzoloMStiglianoAChiodinilltalian Association of Clinical Endocrinologists. AME position statement on adrenal incidentaloma. Eur J Endocrinol2011;164:851-70. doi:10.1530/EJE-10-114721471169

3 MansmannGLauJBalkERothbergMMiyachiYBornsteinSR. The clinically inapparent adrenal mass: update in diagnosis and management. Endocr Rev2004;25:309-40. doi:10.1210/er.2002-003115082524

4 FassnachtMArltWBancosI. Management of adrenal incidentalomas: European Society of Endocrinology clinical practice guideline in collaboration with the European Network for the Study of Adrenal Tumors. Eur J Endocrinol2016;175:G1-34. doi:10.1530/EJE-16-046727390021

5 GrumbachMMBillerBMKBraunsteinGD. Management of the clinically inapparent adrenal mass ("incidentaloma"). Ann Intern Med2003;138:424-9. doi:10.7326/0003-4819-138-5-200303040-0001312614096

6 KloosRTGrossMDFrancisIRKorobkinMShapiroB. Incidentally discovered adrenal masses. Endocr Rev1995;16:460-84.8521790

7 BarzonLSoninoNFalloFPaluGBoscaroM. Prevalence and natural history of adrenal incidentalomas. Eur J Endocrinol2003;149:273-85. doi:10.1530/eje.0.149027314514341

8 BovioSCataldiAReimondoG. Prevalence of adrenal incidentaloma in a contemporary computerized tomography series. J Endocrinol Invest2006;29:298-302. doi:10.1007/BF0334409916699294

9 MayerSKOlignyLLDealCYazbeckSGagnéNBlanchardH. Childhood adrenocortical tumors: case series and reevaluation of prognosis--a 24-year experience. J Pediatr Surg1997;32:911-5. doi:10.1016/S0022-3468(97)90649-79200099

10 YoungWFJr. Clinical practice. The incidentally discovered adrenal mass. N Engl $\mathrm{J}$ Med2007;356:601-10. doi:10.1056/NEJMcp06547017287480

11 SuttonMGShepsSGLieJT. Prevalence of clinically unsuspected pheochromocytoma. Review of a 50-year autopsy series. Mayo Clin Proc1981;56:354-60.6453259

12 ZeigerMAThompsonGBDuhQYAmerican Association of Clinical EndocrinologistsAmerican Association of Endocrine Surgeons. The American Association of Clinical Endocrinologists and American Association of Endocrine Surgeons medical guidelines for the management of adrenal incidentalomas. Endocr Pract2009;15(Suppl 1):1-20 doi:10.4158/EP.15.S1.119632967

13 KapoorAMorrisTRebelloR. Guidelines for the management of the incidentally discovered adrenal mass. Can Urol Assoc J2011;5:241-7. doi:10.5489/cuaj.1113521801680

14 ManteroFTerzoloMArnaldiGStudy Group on Adrenal Tumors of the Italian Society of Endocrinology. A survey on adrenal incidentaloma in Italy. $\mathrm{J}$ Clin Endocrinol Metab2000;85:637-44.10690869

$15 \mathrm{NIH}$ state-of-the-science statement on management of the clinically inapparent adrena mass ("incidentaloma"). NIH Consens State Sci Statements2002;19:1-25.14768652

16 BarzonLScaroniCSoninoNFalloFPaolettaABoscaroM. Risk factors and long-term follow-up of adrenal incidentalomas. J Clin Endocrinol Metab1999;84:520-6.10022410 
17 ElseTKimACSabolchA. Adrenocortical carcinoma. Endocr Rev2014;35:282-326. doi:10.1210/er.2013-102924423978

18 CaoiliEMKorobkinMFrancisIR. Adrenal masses: characterization with combined unenhanced and delayed enhanced CT. Radiology2002;222:629-33. doi:10.1148/radiol.222301076611867777

19 HaiderMAGhaiSJhaveriKLockwoodG. Chemical shift MR imaging of hyperattenuating $(>10 \mathrm{HU})$ adrenal masses: does it still have a role?Radiology2004;231:711-6. doi:10.1148/radiol.231303067615118113

20 BechererAVierhapperHPötziC. FDG-PET in adrenocortical carcinoma. Cancer Biother Radiopharm2001;16:289-95. doi:10.1089/10849780175313136311602999

21 YunMKimWAInafisiNLacorteLJangSAlaviA. 18F-FDG PET in characterizing adrenal lesions detected on CT or MRI. J Nucl Med2001;42:1795-9.11752075

22 ShinYRKimKA. Imaging features of various adrenal neoplastic lesions on radiologic and nuclear medicine imaging. AJR Am J Roentgenol2015;205:554-63. doi:10.2214/AJR.15.1446726295641

23 ChangCAPattisonDATothillRW. (68)Ga-DOTATATE and (18)F-FDG PET/CT in Paraganglioma and Pheochromocytoma: utility, patterns and heterogeneity. Cancer Imaging2016;16:22. doi:10.1186/s40644-016-0084-227535829

24 BancosITamhaneSShahM. DIAGNOSIS OF ENDOCRINE DISEASE: The diagnostic performance of adrenal biopsy: a systematic review and meta-analysis. Eur J Endocrinol2016;175:R65-80. doi:10.1530/EJE-16-029727257146
25 ArltWBiehIMTaylorAE. Urine steroid metabolomics as a biomarker tool for detecting malignancy in adrenal tumors. J Clin Endocrinol Metab2011;96:3775-84. doi:10.1210/jc.2011-156521917861

26 Dennedy MC, Annamalai AK, Prankerd Smith O, et al. Low DHEAS: a sensitive and specific test for detection of subclinical hypercortisolism in adrenal incidentalomas. J Clin Endocrinol Metab 2016;jc.2016-2718.

27 FunderJWCareyRMManteroF. The management of primary aldosteronism: case detection, diagnosis, and treatment: An Endocrine Society clinical practice guideline. J Clin Endocrino Metab2016;101:1889-916. doi:10.1210/jc.2015-406126934393

28 LendersJWMDuhQ-YEisenhoferGEndocrine Society. Pheochromocytoma and paraganglioma: an endocrine society clinical practice guideline. J Clin Endocrino Metab2014;99:1915-42. doi:10.1210/jc.2014-149824893135

29 AdachiJHiraiYTeruiK. A report of 7 cases of adrenal tumors secreting both cortisol and aldosterone. Intern Med2003;42:714-8. doi:10.2169/internalmedicine.42.71412924498

30 BaertDNobelsFVan CrombruggeP. Combined Conn's and Cushing's syndrome: an unusual presentation of adrenal adenoma. Acta Clin Belg1995;50:310-3. doi:10.1080/17843286.1995.117184688533534

Published by the BMJ Publishing Group Limited. For permission to use (where not already granted under a licence) please go to http://group.bmj.com/group/rights-licensing/ permissions 


\section{Tables}

Table 1| Percentage of the various subtypes of adrenal incidentalomas, based on pooled data from several series 12710

Type

Non-functional

Functional (hormonally active)
Subtype

Apparent non-functioning adenoma

\begin{tabular}{lcc} 
Non-functional & Apparent non-functioning adenoma & $71.2-82.4$ \\
\hline \multirow{2}{*}{ Functional (hormonally active) } & Subclinical (autonomous cortisol secretion) Cushing's syndrome & $5.3-7.9$ \\
\cline { 2 - 3 } & Pheochromocytoma & $5.1-5.6$ \\
\cline { 2 - 3 } & Aldosterone producing adenoma & $1.0-1.2$ \\
\hline \multirow{2}{*}{ Malignant } & Adrenocortical cancer & $4.4-4.7$ \\
\cline { 2 - 3 } & Metastatic cancer & $2.1-2.5$ \\
\hline
\end{tabular}

Percentage of adrenal incidentalomas 


\section{Table 2/ Summary of key recommendations from recent European guidance on managing patients with a finding of an incidental adrenal} tumour

\section{Criterion}

General recommendations

\section{European guidance (2016)}

Recommendations for discussion of adrenal incidentalomas in MDT (any of the following): Imaging not consistent with a benign lesion Evidence of hormone excess (including autonomous cortisol secretion)

Evidence of substantial tumour growth during follow-up imaging (more than $20 \%$, or at least a $5 \mathrm{~mm}$ increase in maximum diameter during the following 6-12 months)

Adrenal surgery could be a consideration.

Non-contrast computed tomography with determination of mass density to assess for malignancy. One mg ODST and plasma/urinary metanephrines in all patients. ARR in all patients with concomitant hypertension and/or unexplained hypokalaemia. Sex hormones and steroid precursors in patients with clinical or imaging features of ACC

Surgical recommendation for subclinical Cushing syndrome

Clinical and biochemical assessment for cortisol production and co-morbidities. If worsening then consider surgery

Indications for consideration for surgical intervention

Unilateral adrenal tumour with clinically significant hormone excess Suspicion of malignancy

Specialist screening test for autonomous cortisol production/subclinical Cushing's syndrome

Diagnostic cut-off values for subclinical Cushing's syndrome

$1 \mathrm{mg}$ ODST

\section{Following $1 \mathrm{mg}$ ODST:}

If cortisol between 51 and 138 nmol/L: "possible autonomous cortisol secretion."

If cortisol $>138 \mathrm{nmol} / \mathrm{L}$ (and without clinical features of Cushing's): "autonomous cortisol secretion"

Specialist screening test for pheochromocytoma

Specialist screening test for hyperaldosteronism Plasma free or urinary fractionated metanephrines

Specialist screening test for excess sex hormones ARR

Indications for computed tomography guided needle biopsy

In patients with clinical or radiological features of ACC (+steroid precursors)

In the setting of a newly diagnosed adrenal mass and a history of extra-adrenal malignancy:

Lesion hormonally inactive

Lesion has not been conclusively characterised as benign by imaging

Management would be altered by the knowledge of histology

Imaging Non-contrast computed tomography scan to determine whether the mass is homogenous and lipid rich $(<10 \mathrm{HU})$.

If indeterminate on non-contrast computed tomography, imaging with another modality (contrast computed tomography, MRI, or FDG-PET)

Follow-up if not resected

No further imaging if lesion $<4 \mathrm{~cm}$ and clear benign features on imaging studies. Indeterminate mass repeat non-contrast computed tomography or MRI after 6-12 months. Resect if growth by $>20 \%$ in addition to at least a $5 \mathrm{~mm}$ increase in max diameter. If growth below this threshold additional imaging after 6-12 months. No further hormonal investigation if initial evaluation normal unless new clinical signs of endocrine activity or there is worsening of comorbidities-eg, hypertension or diabetes

MDT: multidisciplinary team, ODST: overnight dexamethasone suppression test, ARR: plasma aldosterone (ng/dL)/renin (ng/mL/h) ratio, ACC: adrenocortical carcinoma, SCS: subclinical Cushing's syndrome, HU: Hounsfield units, MRI: magnetic resonance imaging, FDG-PET: 2-deoxy-2-[fluorine-18]fluoro-D-glucose positron emission tomography/computed tomography 


\section{Figures}

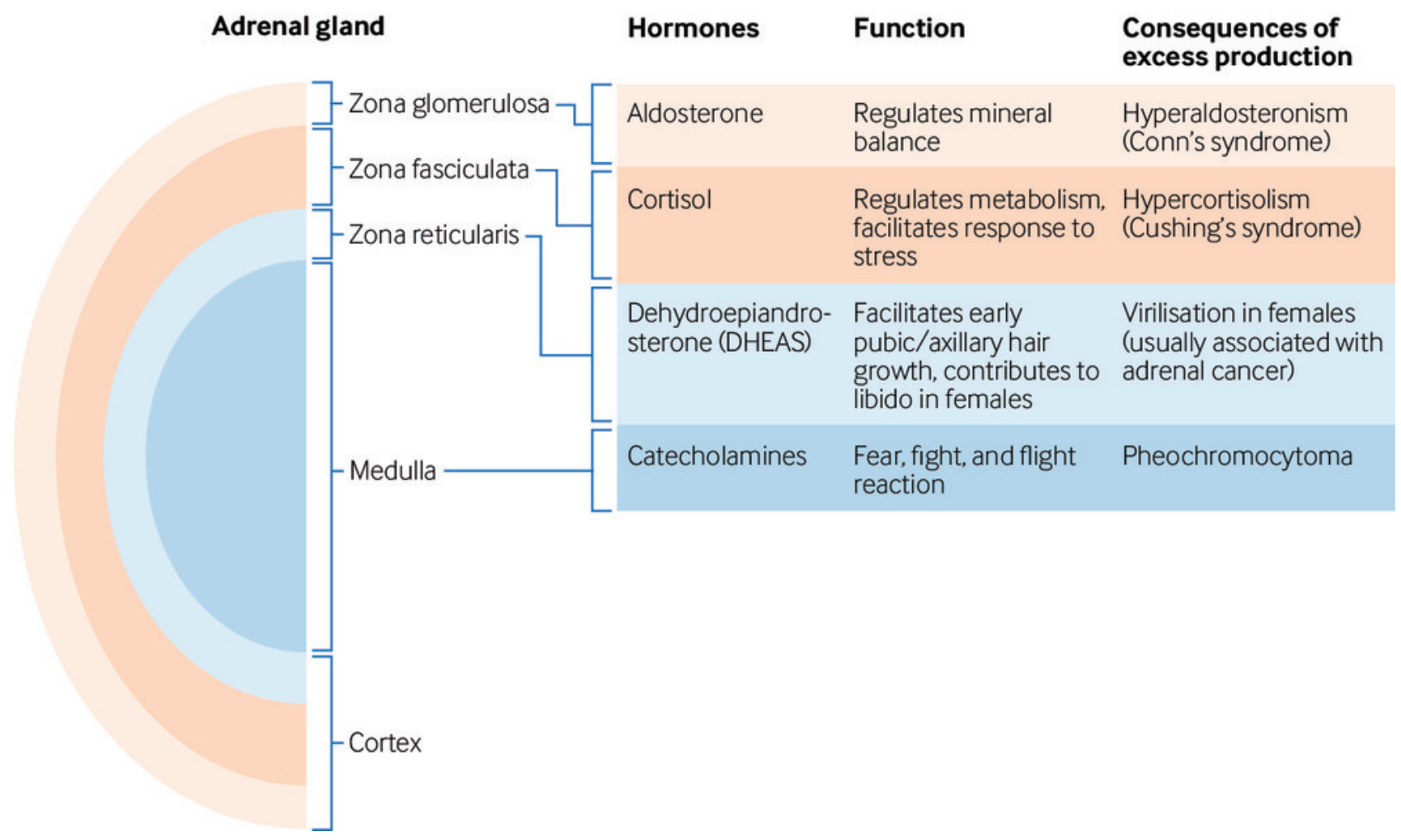

The components of the adrenal gland and consequences of excess hormone production 

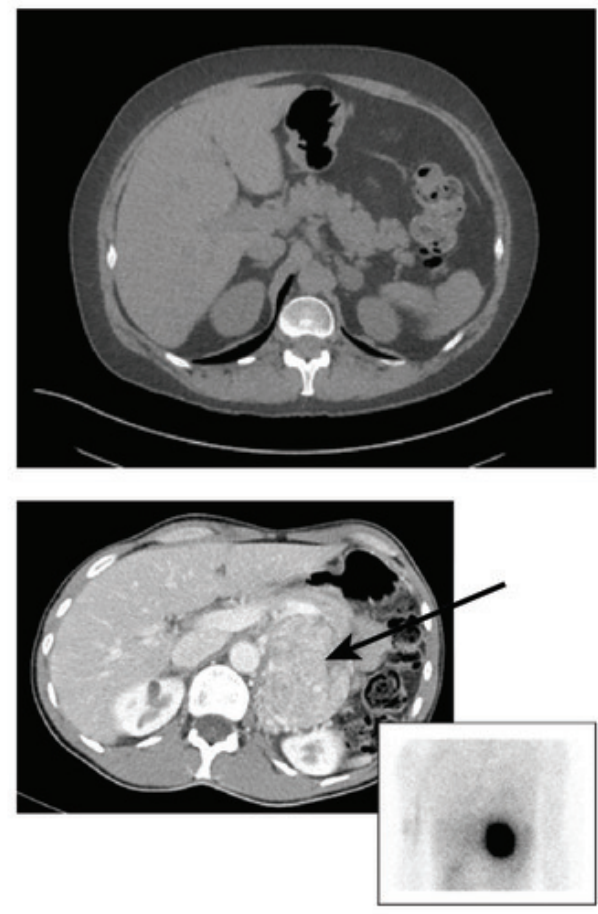

Computed tomography showing lipid-rich and lipid-poor lesions. (a) A $2 \mathrm{~cm}$ left adrenal lesion showing a computed tomography density of less than $10 \mathrm{HU}$, which is suggestive of a lipid-rich adenoma. (b) A $7.7 \mathrm{~cm}$ hypervascular left adrenal mass that is suspicious for pheochromocytoma. Inset shows a metaiodobenzylguanidine scan demonstrating intense uptake in the lesion 